\title{
Auxin response and transport during induction of pedicel abscission in tomato
}

\author{
Xiufen Dong ${ }^{1,2}$, Chao Ma ${ }^{2,3}$, Tao Xu' ${ }^{1}$, Michael S. Reid ${ }^{2 凶}$, Cai-Zhong Jiang $\mathbb{\circledR}^{2,4 凶}$ and Tianlai Li ${ }^{1 凶}$
}

\begin{abstract}
Auxin plays a central role in control of organ abscission, and it is thought that changes in the auxin gradient across the abscission zone are the primary determinant of the onset of abscission. The nature of this gradient, whether in concentration, flow, or perhaps in the response system has not conclusively been determined. We employed a DR5:: GUS auxin response reporter system to examine the temporal and spatial distribution of the auxin response activity in response to developmental and environmental cues during pedicel abscission in tomato. In pedicels of young and fully open flowers, auxin response, as indicated by GUS activity, was predominantly detected in the vascular tissues and was almost entirely confined to the abscission zone (AZ) and to the distal portion of the pedicel, with a striking reduction in the proximal tissues below the AZ - a 'step', rather than a gradient. Following pollination and during early fruit development, auxin response increased substantially throughout the pedicel. Changes in GUS activity following treatments that caused pedicel abscission (flower removal, high temperature, darkness, ethylene, or $\mathrm{N}$-1-naphthylphthalamic acid (NPA) treatment) were relatively minor, with reduced auxin response in the AZ and some reduction above and below it. Expression of genes encoding some auxin efflux carriers (PIN) and influx carriers (AUX/LAX) was substantially reduced in the abscission zone of NPA-treated pedicels, and in pedicels stimulated to abscise by flower removal. Our results suggest that changes in auxin flow distribution through the abscission zone are likely more important than the auxin response system in the regulation of abscission.
\end{abstract}

\section{Introduction}

Abscission is the process of organ separation, which plays a critical role in the plant life cycle ${ }^{1,2}$. Organ shedding occurs at abscission zones (AZ), comprising small, densely cytoplasmic cells at the boundary between an organ and the main plant body ${ }^{3,4}$. Abscission has evolved as a successful strategy to adapt to the environment in response to developmental and environmental cues ${ }^{5}$. Abscission allows plants to detach nonfunctional or diseased organs and is also important for seed dispersal ${ }^{2,6}$. The timing of abscission, especially of flower and fruit abscission, is of interest to agriculture ${ }^{7}$. Breeding of appropriate abscission behavior has successfully solved

Correspondence: Michael S. Reid (msreid@ucdavis.edu) or

Cai-Zhong Jiang (cjiang@ucdavis.edu) or Tianlai Li (tianlaili@126.com)

'Department of Horticulture, Shenyang Agricultural University, Shenyang

110866, China

2Department of Plant Sciences, University of California, Davis, CA 95616, USA

Full list of author information is available at the end of the article crop production and yield problems such as grain shattering, cotton boll shedding, premature legume dehiscence, and mechanical harvest in tomato ${ }^{8}$.

It has been well-demonstrated that the timing of abscission is regulated by cross-talk between the phytohormones auxin and ethylene ${ }^{2}$. Ethylene plays an important role as the regulator that induces cell separation during abscission. Arabidopsis flower abscission is inhibited, for example in the ethylene-insensitive mutants ethylene resistant 1-1 (etr11) and ethylene insensitive 2 (ein 2$)^{9}$. In tomato too, organ abscission is inhibited in ethylene receptor and ethylene sensitivity mutants including EIN (3), Never ripe ( $\mathrm{Nr}$ ), Sletr1-1, and Sletr1-2 $2^{10,11}$.

Auxin plays a critical role in controlling abscission. The consensus of many studies is that the continuous polar flow of auxin passing through the abscission zone (AZ) inhibits abscission and that reduction of this flow initiates abscission by making the AZ sensitive to ethylene, $1,2,12-$ 15. The polar flow is thought to be a reflection of a 
gradient in auxin concentration across the abscission zone. In a series of classic experiments, it was shown that application of indole-3-acetic acid (IAA), to the distal side of Phaseolus vulgaris leaf explants inhibited abscission, while an application to the proximal side accelerated the process ${ }^{16-18}$. The nature of the auxin gradient continues to be the subject of discussion-researchers have proposed that the gradient might be in auxin concentration, auxin biosynthesis, auxin transport, and/or auxin response $\mathrm{e}^{15}$. In Arabidopsis, manipulation of auxin biosynthesis specifically within floral organ AZ demonstrates that reduction of auxin level makes the flower organ shed prematurely ${ }^{7}$. However, the disruption of auxin signaling/ response in AZ delayed the shedding of floral organs ${ }^{7}$, suggesting that a functional IAA signaling/response pathway in AZ cells is required for abscission initiation $^{7,19,20}$. Given the importance of auxin balance between the distal and proximal sides of the AZ for organ shedding $^{16,18}$, it is essential to understand how this auxin gradient is maintained for regulating the initiation of the abscission process in response to developmental and environmental cues.

Environmental cues, especially temperature and light, have huge impacts on organ abscission. It has been reported in various plant species that high temperature accelerates reproductive organ abscission. In cotton, day temperatures above $40^{\circ} \mathrm{C}$ can induce flower abscission ${ }^{19,20}$. In soybean, flower abscission was found to increase with the elevated temperature treatment in three different soybean varieties, while no significant difference was found between control and cool temperature treatments ${ }^{21}$. In addition, the light quality is also critical for organ abscission. Shading, as well as dark treatments, induced reproductive organ abscission in several plants. In pepper, shading treatment enhanced flower abscission in several cultivars $^{22}$. In apple, periods of darkness, shading, or cloudy weather have been showed to increase fruit abscission leading to early fruit drop ${ }^{23,24}$. Nineteen days of shading treatment caused $98 \%$ of the fruit to abscise ${ }^{25}$. In grape, five days of shading at bloom reduced the percentage of fruit $\mathrm{set}^{26}$. However, the mechanisms of high temperature, or dark/low light-induced abscission and whether auxin is involved in these processes are still unknown.

The DR5::GUS reporter system provides a visual indication of the activity of the auxin response in the auxin signal transduction pathway ${ }^{27-29}$. We used tomatoes transformed with this reporter to investigate dynamics of the auxin response system during the different developmental stages and in response to environmental cues in pedicel AZ of tomato and to test the hypothesis that changes in the auxin response system are important in the regulation of abscission.

\section{Results}

The auxin response gradient in tomato pedicels changes during flower development and abscission

To investigate the dynamics of the auxin response system in pedicel during flower development, we collected pedicels two days before anthesis (2 DBA), at anthesis, and 5 and 10 days post anthesis/pollination (5 and 10 DPA). We examined the distribution of the auxin response activity using the DR5::GUS reporter system. GUS activity was concentrated in the vascular tissues, with the majority of activity, particularly in the young flowers and those at anthesis, on the distal side of the abscission zone (Fig. 1a, b), a clear disjunction or 'step' in the auxin response activity at the abscission zone. At anthesis the GUS activity seen in the proximal zone of the younger flowers had disappeared, increasing the difference in the auxin response across the AZ. GUS staining in pedicels at 5 and 10 DPA was considerably enhanced, particularly in the abscission zone and in the vascular tissues of the proximal portion of the pedicel (Fig. 1a, b).

To examine the relationship between these auxin response changes on the control of abscission, we removed flowers $2 \mathrm{DBA}$, at anthesis, and 5 DPA. All of the pedicels whose flowers had been removed at anthesis had abscised $12 \mathrm{~h}$ after flower removal, but there was no abscission of flowers from young and older flowers (Fig. 1c).

\section{Auxin treatment has little effect on the distribution or intensity of the auxin response}

Following flower removal, pedicels were treated at the distal end, or at the junction between the pedicel and the peduncle with lanolin containing $1 \mathrm{mM}$ auxin. Four hours after the start of the experiment there was little obvious change in distribution or intensity of the GUS staining (Fig. S1), indicating that the response system was not rapidly responsive to changes in auxin concentration.

\section{Flower removal results in pedicel abscission, but has little effect on the auxin response pattern}

Eight hours after flower removal pedicels began to separate and most had abscised by $12 \mathrm{~h}$. (Fig. 2a). We tested the changes in the distribution of the auxin response after flower removal using the DR5::GUS reporter system. Four hours after flower removal GUS staining in the pedicels was similar to that in the controls (Fig. 2b). RT-PCR visualization of GUS expression in the tissues confirmed that there was little change in expression in the early stages of the abscission process (Fig. 2c). There was a perceptible decrease in the sharp 'step' in GUS staining across the abscission zone $8 \mathrm{~h}$ after flower 
a
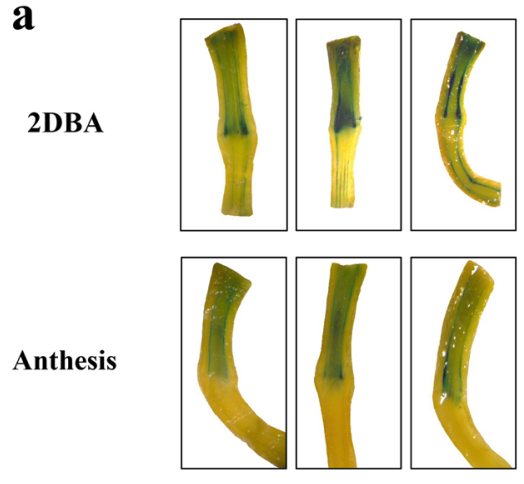

b

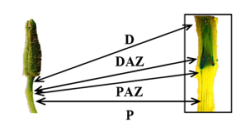

2DBA

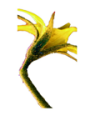

Anthesis

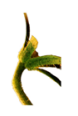

5DPA

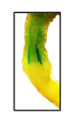

然。
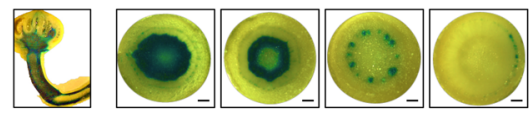

5DPA

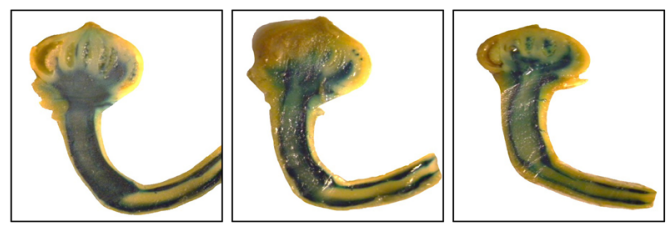

10DPA

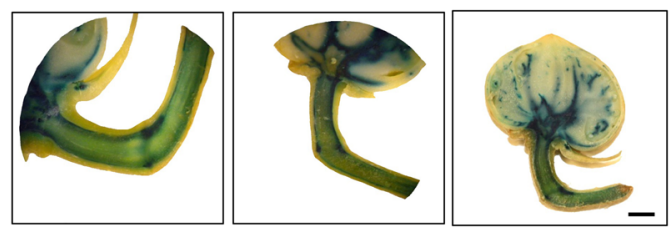

C

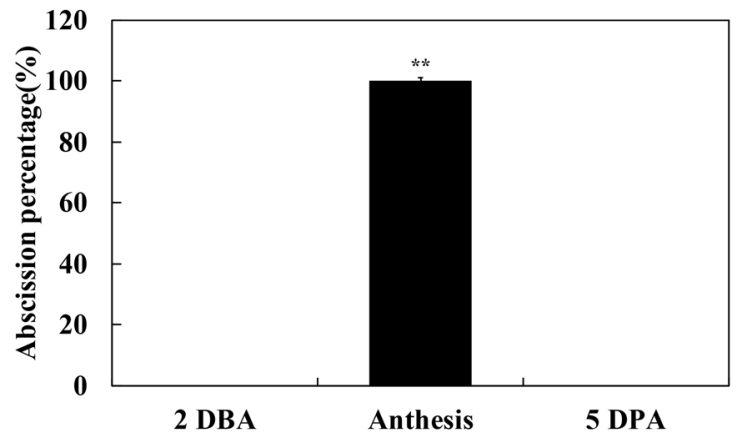

Fig. 1 Changes in auxin response in tomato pedicels and abscission. a Flowers from tomato plants expressing the DR5::GUS auxin response reporter were harvested at different stages before and after anthesis/pollination, and auxin response activity was visualized by staining for GUS activity. Three representative replicates are shown. Scale bars $=200 \mu \mathrm{m}$. b GUS assay in pedicel tissues at different flower development stages. Two days before anthesis (2DBA), at anthesis and 5 days post anthesis/pollination (5DPA), transverse sections were taken from the distal region between the flower and the $A Z(D)$, the distal side of the $A Z$ (DAZ), the proximal side of $A Z(P A Z)$, and the proximal region between the $A Z$ and the peduncle (P). Scale bars $=500 \mu \mathrm{m}$. c Abscission at different flower development stages. Abscission percentage was recorded $12 \mathrm{~h}$ after flower removal. Results are the means of three replicates (15 flowers per replicate) \pm SD. ${ }^{*} p<0.05,{ }^{* *} p<0.01, t$-test

removal, and even more in pedicels that had not yet abscised $12 \mathrm{~h}$ after flower removal, suggesting a reduction in auxin response adjacent to the abscission zone in the later stages of abscission. GUS expression in pedicels $16 \mathrm{~h}$ after flower removal was confined to the distal portion of the pedicel (Fig. 2d).

\section{Inhibition of auxin transport accelerates pedicel} abscission, but has a little immediate effect on the auxin response pattern

Treatment with the auxin transport inhibitor (NPA) mimicked the effect of flower removal on pedicel abscission. Eight hours after the treatment pedicels began to abscise and most had abscised by $12 \mathrm{~h}$ (Fig. 2a). We tested the changes in the distribution of the auxin response after NPA treatment using the DR5:: GUS reporter system. Four hours after flower removal GUS staining in the pedicels was similar to that in the controls (Fig. 2b, d). There was a perceptible decrease in the sharp 'step' in GUS staining across the abscission zone $8 \mathrm{~h}$ after flower removal (Fig. 2d), suggesting a reduction in auxin response adjacent to the abscission zone in the later stages of abscission.

\section{Auxin response changes following flower removal are unaffected by ethylene or inhibition of ethylene action}

Treatment with $10 \mathrm{ppm}$ ethylene accelerated flower abscission following flower removal (Fig. 3a), while pretreatment for $24 \mathrm{~h}$ with 1 -methylcyclopropene completely inhibited abscission (data not shown). GUS activity in ethylene- and 1-MCP-treated pedicels 4 and $8 \mathrm{~h}$ after flower removal showed similar patterns to those seen in the controls (Figs. 2, 3b). The sharp reduction in activity at the abscission zone showed little change $4 \mathrm{~h}$ after flower removal, even in ethylene-treated pedicels that had already abscised but was somewhat reduced after $8 \mathrm{~h}$ both in ethylene-treated and in 1-MCP-treated pedicels (Fig. $3 b, c)$. 


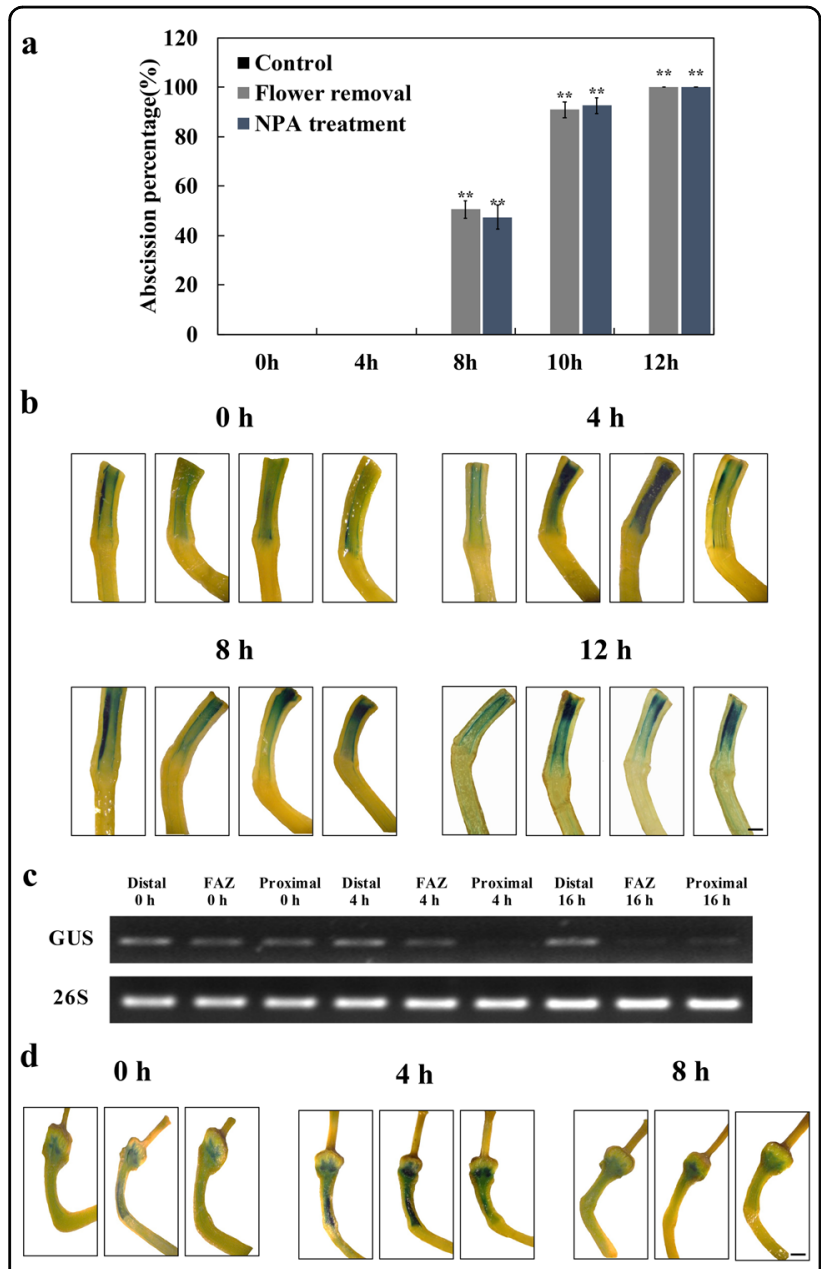

Fig. 2 Effects of flower removal or NPA treatment on the auxin response and pedicel abscission in tomato. a Inflorescences from tomato plants were harvested and placed in water (control) with flowers at anthesis excised or in a solution of $25 \mu \mathrm{M}$ NPA. The percentage of pedicels that abscised was determined at intervals. Results are the means of three replicates ( $>15$ pedicels per replicate) \pm SD. ${ }^{*} p<0.05,{ }^{* *} p<0.01, t$-test. b Flowers from tomato plants expressing the DR5::GUS auxin response reporter were removed at anthesis. Pedicels were harvested at intervals, and the auxin response activity in the pedicel was visualized by staining for GUS activity. Four representative replicates are shown. Scale bars $=200 \mu \mathrm{m}$. c Expression of the gene encoding GUS in distal, abscission zone (FAZ), and proximal portions of pedicels at flower removal and after $4 \mathrm{~h}$ was visualized using RT-PCR. $\mathbf{d}$ Inflorescences from tomato plants expressing the DR5::GUS auxin response reporter were harvested and placed in $25 \mu \mathrm{M}$ NPA. Flowers and their subtending pedicels were harvested at intervals, and the auxin response activity was visualized by staining for GUS activity. Three representative replicates are shown. Scale bars $=200 \mu \mathrm{m}$

Environmental factors that accelerate pedicel abscission have only minor effects on the pattern of the auxin response

When tomato inflorescences were placed at high temperature or in the dark, most flowers abscised after 3 or

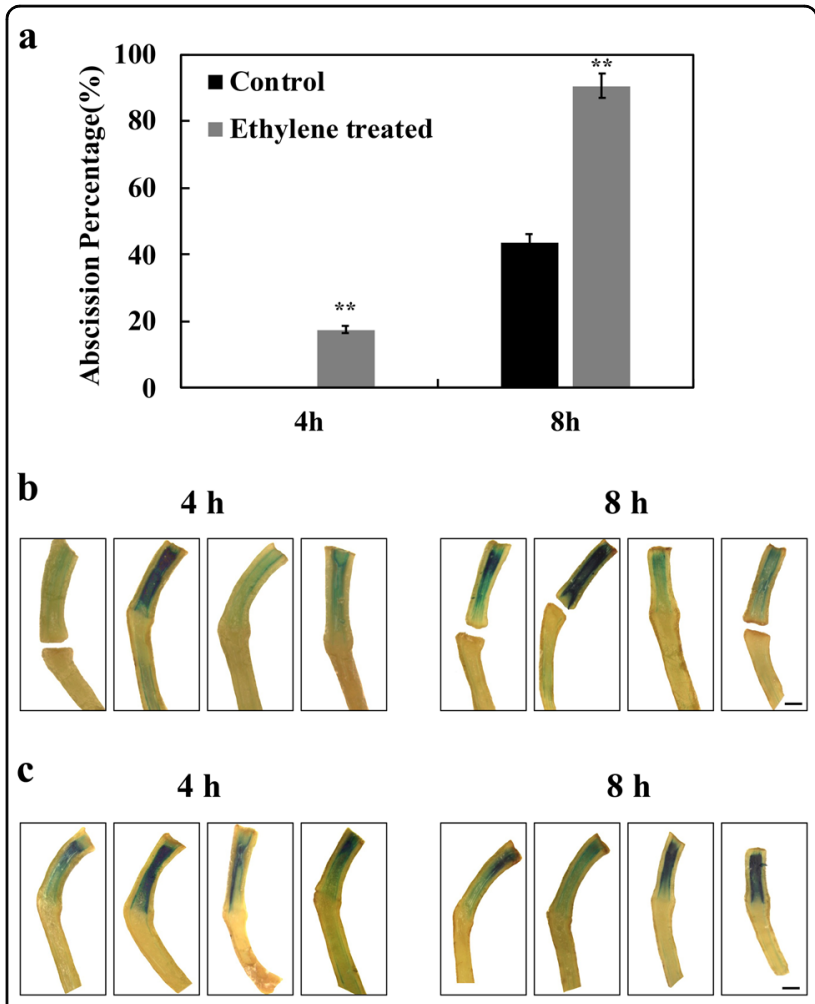

Fig. 3 Effects of ethylene and 1-MCP on flower pedicel abscission and GUS staining in DR5::GUS plants. a At anthesis, flowers were removed from inflorescences of plants bearing the DR5::GUS auxin response reporter and placed in air or $10 \mu \mathrm{L} / \mathrm{L}$ ethylene. Abscission of the pedicels was monitored at intervals. Results are the means of three replicates $(n>15) \pm$ SD. ${ }^{*} p<0.05,{ }^{* *} p<0.01, t$-test. No abscission was observed in pedicels pre-treated with 1-MCP for $24 \mathrm{~h}$. prior to flower removal (data not shown). b GUS activity in ethylene-treated pedicels 4 and $8 \mathrm{~h}$ after flower removal. Four representative replicates are shown. Scale bars $=200 \mu \mathrm{m}$. c GUS activity in 1-MCP-treated pedicels 4 and $8 \mathrm{~h}$ after flower removal. Four replicates are shown. Scale bars $=$ $200 \mu \mathrm{m}$

4-5 days respectively (Fig. 4a, b). The intensity and distribution of the auxin response, as shown by GUS activity in these DR5::GUS tomato plants, was little affected by these substantial changes in environmental conditions. After two days in the dark, for example, the distribution of GUS staining was similar to that in control pedicels when the flowers were at anthesis (Figs. 1 and 4c, d).

\section{Changes in expression of some genes encoding auxin influx and efflux carriers are correlated with abscission}

To determine a possible role for the changes in auxin transport in the control of abscission, we analyzed the expression of genes related to auxin transport, including the pin-formed efflux carriers (PIN) and auxin resistant/ like aux1 (AUXLAX). influx carriers. The changes in expression following flower removal varied among members of the two carrier types (Fig. 5a). By $4 \mathrm{~h}$ after excision, the abundance of transcripts of PIN1, PIN4, 

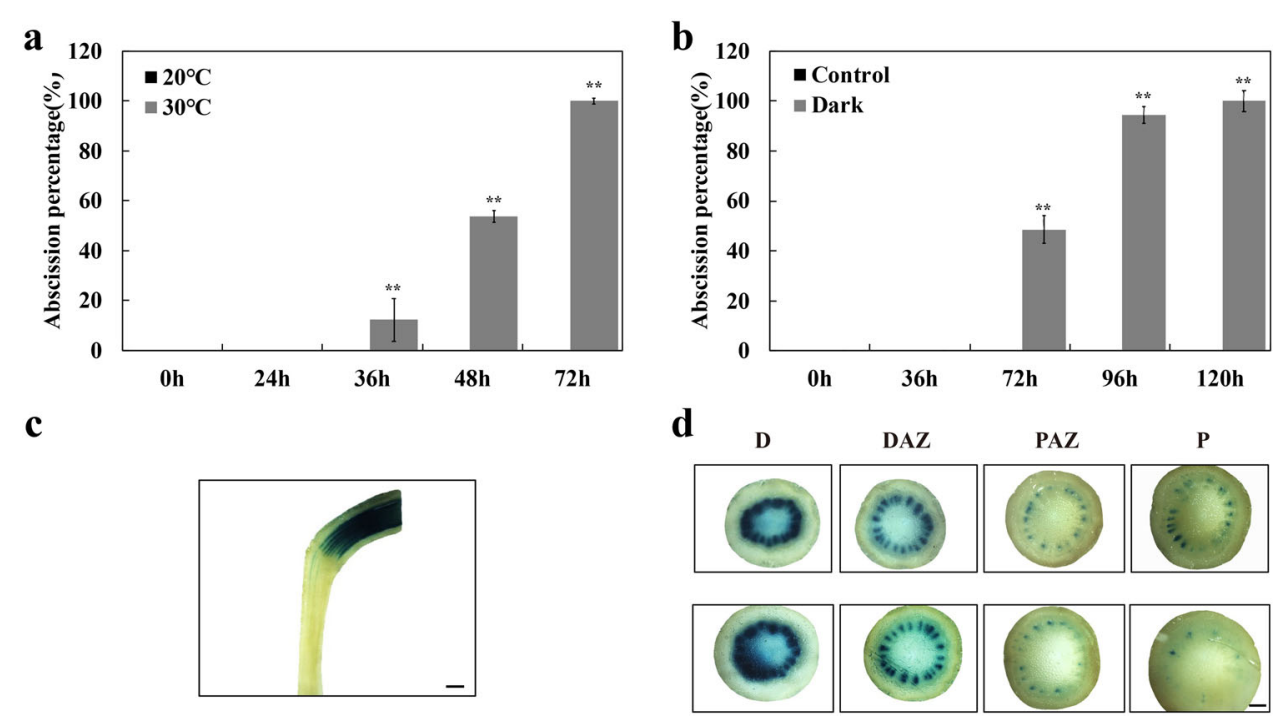

Fig. 4 Abscission and auxin response in response to high temperature or darkness. Inflorescences from tomato plants transformed with the DR5::GUS reporter transgene were harvested and placed in water at $20^{\circ} \mathrm{C}$ (control) in water at $30^{\circ} \mathrm{C}$ (high-temperature treatment) or in water in the dark. $\mathbf{a}, \mathbf{b}$ The percentage of pedicels that abscised was determined at intervals. Results are the means of three replicate inflorescences ( $>15$ pedicels per replicate) $\pm S D .{ }^{*} p<0.05,{ }^{* *} p<0.01, t$-test. Replicate pedicels were stained for GUS activity, shown in a longitudinal section (c), and in transverse sections (d) cut from the distal part of the pedicel (D), from the distal side of the AZ (DAZ), the proximal side of the AZ (PAZ), and from the proximal part of the pedicel (P). Two groups of sections from duplicate pedicels are shown. Scale bars of (c) and (d) are 200 and $500 \mu m$ respectively

PIN9, and $A U X / L A X 2$ was substantially reduced. In contrast, the abundance of $A U X / L A X 1$ was unaffected, and there appeared to be a marked increase in the abundance of transcripts of $A U X / L A X 3$ and $A U X / L A X 4$.

The changes in auxin transport genes induced by treatment with $25 \mu \mathrm{M} \mathrm{N}$-1-naphthylphthalamic acid (NPA), a known auxin transport inhibitor that also accelerates abscission, were somewhat different from those seen following flower excision (Fig. 5b, c). Although PIN6 expression was reduced throughout the pedicel, expression of PIN1, PIN6, and PIN9 were specifically and markedly reduced in the AZ. A similar specific reduction in expression was seen in $A U X / L A X 2$, but the expression of $A U X / L A X 5$ was reduced throughout the pedicel. As seen following flower removal, other $A U X / L A X$ genes remained at high activity $(A U X / L A X 1)$ or even increased in expression (AUX/LAX3). Other $A U X / L A X$ genes remained at high activity $(A U X / L A X 1)$ or even increased in expression $(A U X / L A X 3)$ in response to NPA treatment.

\section{Discussion}

Auxin is considered to be a key hormone in the initiation of abscission; the accepted model suggests that reduced transport of auxin through the $\mathrm{AZ}$ results in sensitization of the AZ to ethylene, which induces the chain of hydrolytic and other processes that lead to cell separation $^{1,2,5,12,13}$. In a previous study ${ }^{30}$, we demonstrated that a knotted homeobox transcription factor, $\mathrm{KD} 1$, plays a role in abscission, apparently by modulating transport of auxin through the AZ. Silencing KD1 increased auxin in the abscission zone, and microarray analysis suggested that this was associated with the downregulation of auxin efflux transporters, particularly PIN9. The study also suggested that the change in auxin distribution across the abscission zone resulting from KD1 activity was associated with a change in the activity of the auxin response pathway, and the experiments reported here were designed to test that hypothesis.

We found that the auxin response pathway certainly does change during development (Fig. 1). High activity was seen in the distal portion of the pedicel during flower opening, with a marked disjunction or 'step' on the distal side of the AZ. Following pollination, response activity increased substantially, particularly in the young fruit, the $\mathrm{AZ}$, and in the proximal region of the pedicel. Our data did not support the hypothesis that changes in distribution or activity of the auxin response system play an important role in the regulation of abscission. Removing the flowers at anthesis, which induces pedicel abscission within $8 \mathrm{~h}$ (Fig. 2) had little effect on the distribution or activity of auxin response (Fig. 2b), particularly in the early hours after excision, when the abscission process is initiated. The visual results from GUS staining of the pedicels are supported by RT-PCR analysis of the expression of GUS transcripts (Fig. 2c), which shows a marked 'step' in transcript abundance across the abscission zone, and a change in expression pattern only at $12 \mathrm{~h}$ after excision, when most pedicels have already abscised. 


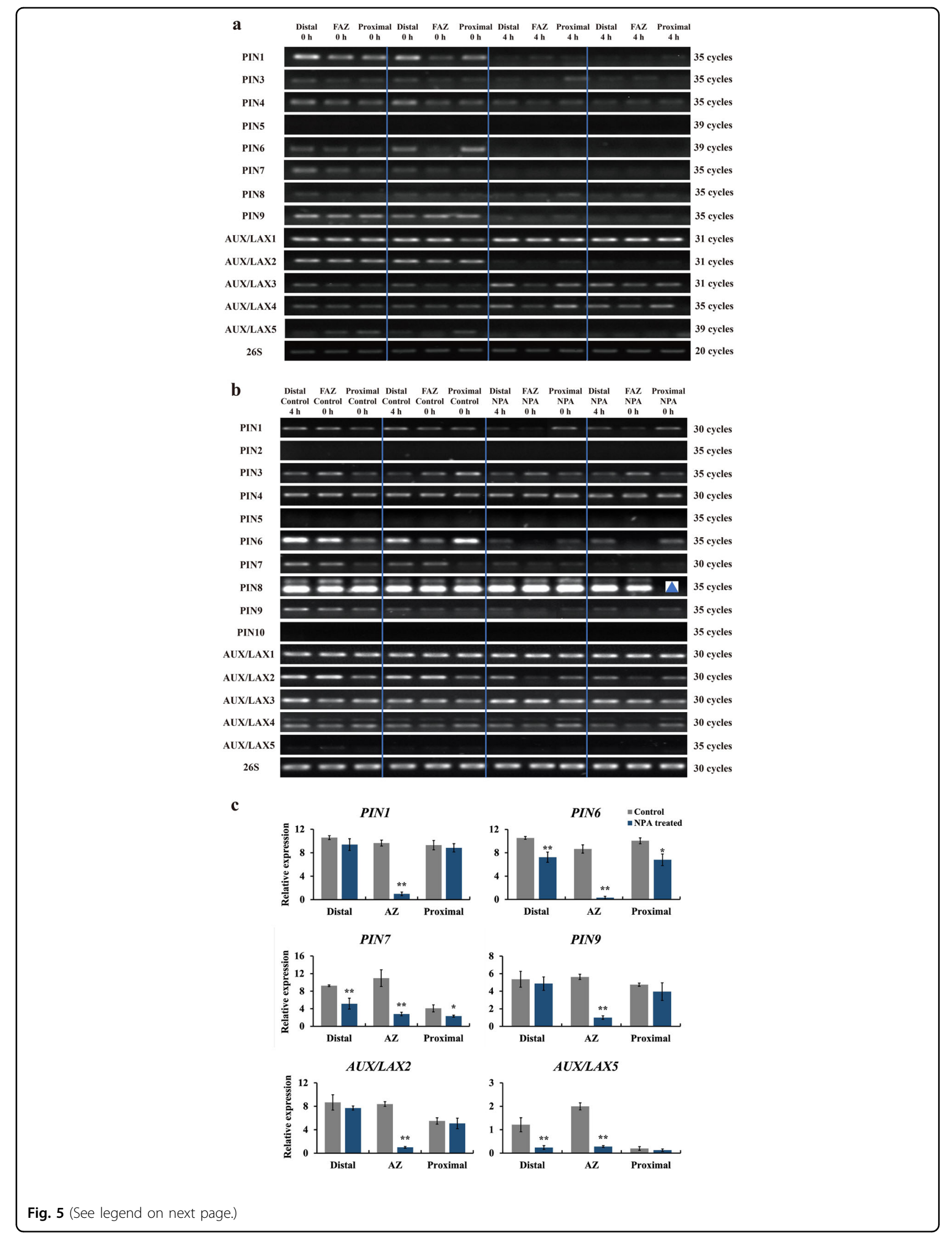


(see figure on previous page)

Fig. 5 Expression pattern of auxin transport-related genes following flower removal and NPA treatment. a Semi-quantitative RT-PCR analyses were carried out to compare transcript levels between pedicels at flower removal (control) and $4 \mathrm{~h}$ later. Samples were taken from the distal side of the pedicel, the abscission zone (AZ), and the proximal side of the pedicel. Representative PCR profiles from duplicate biological samples are shown. Different numbers represent cycles for Semi-Quantitative RT-PCR. Semi-quantitative RT-PCR (b) and real-time quantitative PCR (RT-qPCR) (c) analyses were carried out to compare transcript levels between pedicels following flower removal (control) or treatment with NPA. Samples were taken from the distal side of the flower pedicel, flower abscission zone (FAZ), and the proximal side of the flower pedicel. Representative PCR products from duplicate biological samples are shown. The triangle indicates one missing sample. RT-qPCR results were the means of three biological replicates \pm SD. ${ }^{*} p<0.05,{ }^{* *} p<0.01, t$-test

The conclusion that a change in distribution or activity of the auxin response system plays no regulatory role in pedicel abscission is supported by our additional data. None of the other manipulations that affected the occurrence or timing of abscission had a marked effect on GUS staining. Placement of auxin distal or proximal to the abscission zone, treatment with NPA, treatment with ethylene, or 1-MCP (which inhibits ethylene action), placing inflorescences in the dark, or at high temperature, all had significant and varied effects on abscission, but the distribution of auxin response system as indicated by GUS activity was remarkably stable.

In contrast, the data presented here demonstrated that the early stages of abscission were associated with marked changes in the distribution and activity of genes involved in auxin transport. This is in agreement with our earlier results $^{30}$. Application of the auxin transport inhibitor, NPA, resulted in a marked reduction of expression of genes encoding enzymes involved in auxin transport (Fig. 5b). Particularly striking decreases were seen in the expression of PIN1, PIN6, PIN9, and AUX/LAX2 in the abscission zone itself. This general pattern was also seen following flower removal, although the reduction in expression of the PIN genes appeared to be less tissuespecific (Fig. 5a). These changes are consistent with the observations of Shi et al. ${ }^{31}$, who also found a substantial reduction in SIPIN1 expression following flower removal, and suggested that it might play a role in modulating the auxin content of the AZ. Silencing of SIPIN1 expression accelerated pedicel abscission by simultaneously increasing auxin accumulation in the ovary and decreasing the auxin levels in the $A Z^{31}$, suggesting that auxin transport modulates auxin balance to influence pedicel abscission. Interestingly, PIN2/5/10 were not expressed in pedicels (Fig. 5b). This is different from other reports that downregulation of PIN5 in the flower pedicel reduces intracellular auxin accumulation in the endoplasmic reticulum (ER), which is expected to control auxin availability for auxin signaling/response in the nuclei of $\mathrm{AZ}$ cells $^{32}$. The exact regulatory roles of these auxin transporters in the induction of abscission need further investigation in the future.

Auxin and abiotic stress work together affecting plant growth and development. In Arabidopsis, the shootward auxin transport can be inhibited by the reduction of PIN1/ 3 transcripts under low temperature and increased by the upregulation of PIN2 under high temperature ${ }^{33,34}$. In addition, high temperature induces hypocotyl elongation by regulating PIF4-mediated auxin biosynthesis ${ }^{35,36}$. Our data showed that both high temperature and darkness can accelerate abscission (Fig. 4). However, the intensity and distribution of the auxin response were almost little affected by these substantial changes in environmental conditions (Fig. 4). It is still unknown if auxin transport can affect the pedicel abscission in tomato under these environmental conditions and would require further investigation.

Our results are consistent with a model that places the primary control of abscission on the concentration of auxin in the abscission zone $\mathrm{e}^{1,2,5,12-15}$. Concurrent changes in the relative rates of influx and efflux might plausibly result in marked changes in auxin concentration, triggering the sensitivity to ethylene that results in the onset of the abscission process. Our data indicate that at $4 \mathrm{~h}$ after flower removal, expression of genes encoding auxin efflux enzymes (PIN1,4,6, and 9) fell (Fig. 5) while the expression of genes encoding influx enzymes (AUX/ $L A X 1,3$, and 4) increased. We can imagine a scenario where the activity of KD1 is controlled by auxin transported from the flower. When auxin flow falls, KD1 might modulate the expression of genes involved in auxin influx and efflux, amplifying the effect of small changes in auxin flow, and resulting in a marked fall in auxin content of the abscission zone, triggering the changes that result in separation. In this scenario, the auxin response system is an important factor, but it functions as a reporter of auxin content, and does not rapidly change activity or distribution in response to changes in auxin supply from the flower.

\section{Materials and methods}

\section{Plant materials and treatments}

Tomato (Solanum lycopersicum) germplasm 'VF36' (Accession: LA0490) seeds were provided by the Tomato Genetics Resource Center, University of California Davis. The homozygous DR5::GUS transgenic tomato plants ('VF36' background) were provided by Dr. Neelima Sinha (University of California Davis). 
Tomato inflorescences were harvested at 10 AM from plants grown in the greenhouse at the University of California Davis ${ }^{30}$. Inflorescences with at least two newly opened flowers at anthesis (Day 0), two days before anthesis (2 DBA) and five days post anthesis (5 DPA) were cut on the proximal side of the $\mathrm{AZ}$ and placed in vials, and held in a chamber into which humid air was continuously flown through. For testing abscission triggered by auxin depletion via flower removal, flowers were removed with a sharp razor blade by cutting on the distal side of the AZ, and abscission of the remaining pedicel from the peduncle was monitored at intervals ${ }^{12}$. For testing abscission triggered by auxin transporter inhibitor, N-1naphthylphthalamic acid (NPA), the inflorescences were placed in vials containing $10 \mathrm{ml}$ of $25 \mu \mathrm{M}$ NPA solution. Flowers were not removed for NPA treatments. Control inflorescences were placed in a vial containing a solution of the equivalent concentration of dimethyl sulfoxide ${ }^{30}$. For testing temperature-dependent abscission, the inflorescences were harvested at the anthesis stage. The pedicels with/out flowers were placed in the testing chambers in temperature-controlled rooms with indicated temperatures. For dark-induced pedicel abscission, the inflorescences were harvested at the anthesis stage with/ out flower removal and placed in the chambers under the dark conditions at $20^{\circ} \mathrm{C}$. For testing ethylene-triggered pedicel abscission, the inflorescences were harvested at the anthesis stage with flower removal and placed in the ethylene chambers $(10 \mu \mathrm{L} / \mathrm{L})^{37}$. All the experiments were carried out with at least three biological replicates.

\section{GUS staining}

Tomato inflorescences were fixed in $90 \%(\mathrm{v} / \mathrm{v})$ acetone for $20 \mathrm{~min}$ and then placed into GUS staining buffer (0.5 mM 5-bromo-4-chloro-3-indolyl- $\beta$-glucuronic acid, $0.15 \mathrm{M} \mathrm{NaH}_{2} \mathrm{PO}_{4}(\mathrm{pH} 7), 2 \mathrm{mM} \mathrm{K}_{3} \mathrm{Fe}(\mathrm{CN})_{6}, 2 \mathrm{mM} \mathrm{K}_{4} \mathrm{Fe}$ $(\mathrm{CN})_{6}$, and $0.05 \%(\mathrm{v} / \mathrm{v})$ Triton $\left.\mathrm{X}-100\right)$. The inflorescences were infiltrated in a capped $60 \mathrm{~mL}$ syringe by depressing the plunger for $3 \mathrm{~min}$ and then vacuuming for $1 \mathrm{~h}$. After incubation in the dark at $37{ }^{\circ} \mathrm{C}$ for $16 \mathrm{~h}$, GUS-stained tissues were rinsed and stored in $90 \%(\mathrm{v} / \mathrm{v})$ acetone. Pedicels were separated into distal (D), distal abscission zone (DAZ), proximal abscission zone (PAZ), and proximal $(\mathrm{P})$ sections with a razor blade after staining. Images are representative of $>20$ observed samples stained in three independent experiments ${ }^{30}$.

\section{RNA isolation, semi-quantitative RT-PCR, and quantitative RT-PCR}

Total RNAs were extracted using Trizol reagent following the protocol provided by the manufacturer (Invitrogen) and treated with DNase I (Thermo Scientific). Total RNAs from each sample were used for first-strand cDNA synthesis in a final volume of $20 \mu \mathrm{l}$. For RT-PCR, we used $1 \mu \mathrm{lDNA}$ as the template. For semi-quantitative RTPCR, the number of PCR cycles was optimized for each gene based on the amplification level in the lag phase. The resulting PCR products were analyzed by $1 \%(\mathrm{w} / \mathrm{v})$ agarose gel electrophoresis and recorded with the Gel Logic 200 (Kodak, Rochester, NY). The expression levels of $26 \mathrm{~S}$ ribosomal RNA were used as an internal control to compare the relative gene expression levels for each gene ${ }^{38}$. For real-time quantitative PCR (qRT-PCR), a method with two steps of Takara real-time PCR was used to amplify representative the auxin transport-related genes. The levels of relative gene expression were calculated from $\Delta \Delta \mathrm{Ct}$ values ${ }^{39,40}$. A constitutively expressed actin gene was used as a reference gene to normalize cDNA. Each experiment was performed independently two times with at least three biological samples. The primers used in this study are listed in the Supplementary file.

\section{Acknowledgements}

We thank Dr. Neelima Sinha for providing the DR5::GUS tomato line, the Tomato Genetics Resource Center of the University of California for tomato seeds, and Ayla Norris for careful editing of the manuscript. This work was supported by the National Key Research and Development Program of China (2018YFD1000800), the National Natural Science Foundation of China (31991184, 31672197), and China Scholarship Council.

\section{Author details}

1 Department of Horticulture, Shenyang Agricultural University, Shenyang 110866, China. ${ }^{2}$ Department of Plant Sciences, University of California, Davis, CA 95616, USA. ${ }^{3}$ Department of Ornamental Horticulture, College of Horticulture, China Agricultural University, Beijing 100193, China. ${ }^{4}$ Crops Pathology \& Genetic Research Unit, USDA-ARS, Davis, CA 95616, USA

\section{Author contributions}

M.S.R., C.Z.J., and T.L. conceived and designed the experiments. X.D. and C.M conducted the experiments, analyzed the data with assistance from T.X., and wrote the paper. M.S.R., C.Z.J., C.M., and T.X. revised the paper. All authors reviewed and approved the submission.

\section{Conflict of interest}

The authors declare no competing interests.

Supplementary information The online version contains supplementary material available at https://doi.org/10.1038/s41438-021-00626-8.

Received: 3 February 2021 Revised: 7 June 2021 Accepted: 7 June 2021 Published online: 01 September 2021

References

1. Addicott, F. T. Abscission (University of California Press, Berkeley, CA, 1982).

2. Roberts, J. A., Elliott, K. A. \& Gonzalez-Carranza, Z. H. Abscission, dehiscence, and other cell separation processes. Annu. Rev. Plant Biol. 53, 131-158 (2002).

3. McKim, S. M. et al. The BLADE-ON-PETIOLE genes are essential for abscission zone formation in Arabidopsis. Development 135, 1537-1546 (2008).

4. Tucker, M. \& Kim, J. Abscission research: What we know and what we still need to study. Stewart Postharvest Rev. 11, 1-7, https://doi.org/10.2212/ spr.2015.2.1 (2015).

5. Taylor, J. E. \& Whitelaw, C. A. Signals in abscission. N. Phytologist 151, 323-339 (2001).

6. Bleecker, A. B. \& Patterson, S. E. Last exit: senescence, abscission, and meristem arrest in Arabidopsis. Plant Cell 9, 1169-1179 (1997). 
7. Basu, M. M. et al. The manipulation of auxin in the abscission zone cells of arabidopsis flowers reveals that indoleacetic acid signaling is a prerequisite for organ shedding. Plant Physiol. 162, 96-106 (2013).

8. Nakano, T. \& Ito, Y. Molecular mechanisms controlling plant organ abscission. Plant Biotechnol. 30, 209-216 (2013).

9. Patterson, S. E. \& Bleecker, A. B. Ethylene-dependent and -independent processes associated with floral organ abscission in Arabidopsis. Plant Physiol. 134, 194-203 (2004).

10. Lanahan, M. B., Yen, H. C., Giovannoni, J. J. \& Klee, H. J. The never ripe mutation blocks ethylene perception in tomato. Plant Cell 6, 521-530 (1994).

11. Okabe, Y. et al. Tomato TILLING technology: development of a reverse genetics tool for the efficient isolation of mutants from micro-tom mutant libraries. Plant Cell Physiol. 52, 1994-2005 (2011).

12. Abeles, F. B. \& Rubinstein, B. Regulation of ethylene evolution and leaf abscission by auxin. Plant Physiol. 39, 963-969 (1964).

13. Meir, S., Hunter, D. A., Chen, J. C., Halaly, V. \& Reid, M. S. Molecular changes occurring during acquisition of abscission competence following auxin depletion in Mirabilis jalapa. Plant Physiol. 141, 1604-1616 (2006).

14. Meir, S. et al. Microarray analysis of the abscission-related transcriptome in the tomato flower abscission zone in response to auxin depletion. Plant Physiol. 154, 1929-1956 (2010).

15. Ma, C., Jiang, C.-Z. \& Gao, J. Regulatory Mechanisms Underlying Activation of Organ Abscission. Annu. Plant Rev. 4, 27-56, https://doi.org/10.1002/ 9781119312994.apr0741 (2021).

16. Addicott, F. T., Lynch, R. S. \& Carns, H. R. Auxin gradient theory of abscission regulation. Science 121, 644-645 (1955).

17. Jacobs, W. P., Shield, J. A. \& Osborne, D. J. Senescence factor \& abscission of coleus leaves. Plant Physiol. 37, 104-106 (1962).

18. Louie, D. S. J. \& Addicott, F. T. Applied auxin gradients and abscission in explants. Plant Physiol. 45, 654-657 (1970).

19. Najeeb, U., Sarwar, M., Atwell, B. J., Bange, M. P. \& Tan, D. K. Y. Endogenous ethylene concentration is not a major determinant of fruit abscission in heatstressed cotton (Gossypium hirsutum L.). Front. Plant Sci. 8, 1615 (2017).

20. Reddy, V. R., Baker, D. N. \& Hodges, H. F. Temperature effects on cotton canopy growth, photosynthesis, and respiration. Agron. J. 83, 699-704 (1991).

21. Baten, M. A., Khatun, R., Khan, M. M. K., Hassan, M. M. \& Hoque, A. Effect of temperature on flower and pod abscission and yield of three soybean genotypes. J. Environ. Sci. Nat. Resour. 8, 89-92 (2016).

22. Aloni, B., Karni, L. Z., Zaidman, Z. \& Schaffer, A. A. Changes of carbohydrates in pepper (Capsicum annuum L.) flowers in relation to their abscission under different shading regimes. Ann. Bot. 78, 163-168 (1996).

23. Byers, R. E., Carbaugh, D. H., Presley, C. N. \& Wolf, T. K. The influence of low light on apple fruit abscission. J. Horticultural Sci. 66, 7-17 (1991).

24. Byers, R. E. Influence of temperature and darkness on apple fruit abscission and chemical thinning. J. Tree Fruit. Prod. 3, 41-53 (2002).
25. Zhu, H. et al. Transcriptomics of shading-induced and NAA-induced abscission in apple (Malus Domestica) reveals a shared pathway involving reduced photosynthesis, alterations in carbohydrate transport and signaling, and hormone crosstalk. BMC Plant Biol. 11, 138 (2011).

26. Ferree, D. C., McArtney, S. J. \& Scurlock, D. M. Influence of irradiance and period of exposure on fruit set of French-American hybrid grapes. J. Am. Soc. Horticultural Sci. 126, 283 (2001).

27. Ulmasov, T., Murfett, J., Hagen, G. \& Guilfoyle, T. J. Aux/IAA proteins repress expression of reporter genes containing natural and highly active synthetic auxin response elements. Plant Cell 9, 1963-1971 (1997).

28. Koenig, D., Bayer, E., Kang, J., Kuhlemeier, C. \& Sinha, N. Auxin patterns Solanum lycopersicum leaf morphogenesis. Development 136, 2997-3006 (2009)

29. Dubreuil, C., Jin, X., Grönlund, A. \& Fischer, U. A local auxin gradient regulates root cap self-renewal and size homeostasis. Curr. Biol. 28, 2581-2587 (2018).

30. Ma, C. et al. A KNOTTED1-LIKE HOMEOBOX protein regulates abscission in tomato by modulating the auxin pathway. Plant Physiol. 167, 844-853 (2015).

31. Shi, Z. et al. SIPIN1 regulates auxin efflux to affect flower abscission process. Sci. Rep. 7, 14919 (2017)

32. Meir, S., Sundaresan, S., Riov, J., Agarwal, I. \& Philosoph-Hadas, S. Role of auxin depletion in abscission control. Stewart Postharvest Rev. 11, 1-15 (2015).

33. Hanzawa, T. et al. Cellular Auxin homeostasis under high temperature is regulated through a SORTING NEXIN1-dependent endosomal trafficking pathway. Plant Cell 25, 3424-3433 (2013).

34. Zhu, J. et al. Low temperature inhibits root growth by reducing auxin accumulation via ARR1/12. Plant Cell Physiol. 56, 727-736 (2014).

35. Franklin, K. A. et al. PHYTOCHROME-INTERACTING FACTOR 4 (PIF4) regulates auxin biosynthesis at high temperature. Proc. Natl Acad. Sci. USA 108, 20231-20235 (2011)

36. Sun, J., Qi, L., Li, Y., Chu, J. \& Li, C. PIF4-mediated activation of YUCCA8 expression integrates temperature into the auxin pathway in regulating Arabidopsis hypocotyl growth. PLoS Genet. 8, e1002594 (2012).

37. Jiang, C. Z., Lu, F., Imsabai, W., Meir, S. \& Reid, M. S. Silencing polygalacturonase expression inhibits tomato petiole abscission. J. Exp. Bot 59, 973-979 (2008).

38. Estrada-Melo, A. C., Chao, Reid, M. S. \& Jiang, C. Z. Overexpression of an ABA biosynthesis gene using a stress-inducible promoter enhances drought resistance in petunia. Horticulture Res. 2, 15013 (2015).

39. Livak, K. J. \& Schmittgen, T. D. Analysis of relative gene expression data using real-time quantitative PCR and the 2(-Delta Delta $C(T)$ ) Method. Methods 25 402-408 (2001).

40. Yin, D. et al. PhERF2, an ethylene-responsive element binding factor, plays an essential role in waterlogging tolerance of petunia. Horticulture Res. 6, 83 (2019). 